STATE OF ALASKA

DEPARTMENT OF NATURAL RESOURCES

Alaska Geologic Materials Center Data Report No. 403

\title{
No. 403
}

Full Metal Minerals and Calista Corporation, 2012, Borehole inventory, assay results, drilling data, lithologic descriptions, and Microsoft Access file for the Russian Mountains Prospect (Owhat, Louise, and Headwall) Aniak Mining District, Alaska

Zip file available for download

Received June 2012

All data reports may be downloaded free of charge from the DGGS website. 


\section{Alaska GMC Russian Mountains Prospect Inventory}

\begin{tabular}{|c|c|c|c|c|c|c|c|c|}
\hline prospect_name & ehole_na & company & box & interval_top & interval_bottom & core_diameter & box_type & sample_remarks \\
\hline Russian Mountains (Owhat) & RM-01 & FMM & 1 & 0 & $\begin{array}{r}9.5 \\
\end{array}$ & $47.6 / 1.9(\mathrm{~mm} / \mathrm{in})-\mathrm{N}$ core & $N X$ & \\
\hline Russian Mountains (Owhat) & RM-01 & FMM & 2 & 9.5 & 19 & $47.6 / 1.9(\mathrm{~mm} / \mathrm{in})$ - N core & NX & \\
\hline Russian Mountains (Owhat) & RM-01 & FMM & 3 & 19 & 28 & $47.6 / 1.9(\mathrm{~mm} / \mathrm{in})$ - N core & NX & \\
\hline Russian Mountains (Owhat) & RM-01 & FMM & 4 & 28 & 38 & $47.6 / 1.9(\mathrm{~mm} / \mathrm{in})$ - N core & NX & \\
\hline Russian Mountains (Owhat) & RM-01 & FMM & 5 & 38 & 47 & $47.6 / 1.9(\mathrm{~mm} / \mathrm{in})$ - N core & NX & \\
\hline Russian Mountains (Owhat) & RM-01 & FMM & 6 & 47 & 57 & $47.6 / 1.9(\mathrm{~mm} / \mathrm{in})$ - N core & NX & \\
\hline Russian Mountains (Owhat) & RM-01 & FMM & 7 & 57 & 66 & $47.6 / 1.9(\mathrm{~mm} / \mathrm{in})$ - N core & NX & \\
\hline Russian Mountains (Owhat) & RM-01 & FMM & 8 & 66 & 75 & $47.6 / 1.9(\mathrm{~mm} / \mathrm{in})$ - N core & NX & \\
\hline Russian Mountains (Owhat) & RM-01 & FMM & 9 & 75 & 84.5 & $47.6 / 1.9(\mathrm{~mm} / \mathrm{in})$ - N core & NX & \\
\hline Russian Mountains (Owhat) & RM-01 & FMM & 10 & 84.5 & 94 & $47.6 / 1.9(\mathrm{~mm} / \mathrm{in})$ - N core & NX & \\
\hline Russian Mountains (Owhat) & RM-01 & FMM & 11 & 94 & 103.5 & $47.6 / 1.9(\mathrm{~mm} / \mathrm{in})$ - N core & NX & jumbled core \\
\hline Russian Mountains (Owhat) & RM-01 & FMM & 12 & 103.5 & 112.5 & $47.6 / 1.9(\mathrm{~mm} / \mathrm{in})$ - N core & NX & \\
\hline Russian Mountains (Owhat) & RM-01 & FMM & 13 & 112.5 & 121.5 & $47.6 / 1.9(\mathrm{~mm} / \mathrm{in})$ - N core & NX & \\
\hline Russian Mountains (Owhat) & RM-01 & FMM & 14 & 121.5 & 130.5 & $47.6 / 1.9(\mathrm{~mm} / \mathrm{in})$ - N core & NX & \\
\hline Russian Mountains (Owhat) & RM-01 & FMM & 15 & 130.5 & 140.5 & $47.6 / 1.9(\mathrm{~mm} / \mathrm{in})$ - N core & $\mathrm{NX}$ & \\
\hline Russian Mountains (Owhat) & RM-01 & FMM & 16 & 140.5 & 149.5 & $47.6 / 1.9(\mathrm{~mm} / \mathrm{in})$ - N core & NX & \\
\hline Russian Mountains (Owhat) & RM-01 & FMM & 17 & 149.5 & 159 & $47.6 / 1.9(\mathrm{~mm} / \mathrm{in})$ - N core & NX & \\
\hline Russian Mountains (Owhat) & RM-01 & FMM & 18 & 159 & 168 & $47.6 / 1.9(\mathrm{~mm} / \mathrm{in})$ - N core & NX & \\
\hline Russian Mountains (Owhat) & RM-01 & FMM & 19 & 168 & 177.5 & $47.6 / 1.9(\mathrm{~mm} / \mathrm{in})$ - N core & $\mathrm{NX}$ & \\
\hline Russian Mountains (Owhat) & RM-01 & FMM & 20 & 177.5 & 187 & $47.6 / 1.9(\mathrm{~mm} / \mathrm{in})$ - N core & NX & missing box \\
\hline Russian Mountains (Owhat) & RM-01 & FMM & 21 & 187 & 196 & $47.6 / 1.9(\mathrm{~mm} / \mathrm{in})$ - N core & $\mathrm{NX}$ & \\
\hline Russian Mountains (Owhat) & RM-01 & FMM & 22 & 196 & 205 & $47.6 / 1.9(\mathrm{~mm} / \mathrm{in})$ - N core & NX & \\
\hline Russian Mountains (Owhat) & RM-01 & FMM & 23 & 205 & 215 & $47.6 / 1.9(\mathrm{~mm} / \mathrm{in})$ - N core & NX & \\
\hline Russian Mountains (Owhat) & RM-01 & FMM & 24 & 215 & 224 & $47.6 / 1.9(\mathrm{~mm} / \mathrm{in})$ - N core & NX & \\
\hline Russian Mountains (Owhat) & RM-01 & FMM & 25 & 224 & 234 & $47.6 / 1.9(\mathrm{~mm} / \mathrm{in})$ - N core & NX & \\
\hline Russian Mountains (Owhat) & RM-01 & FMM & 26 & 234 & 244 & $47.6 / 1.9(\mathrm{~mm} / \mathrm{in})$ - N core & NX & \\
\hline Russian Mountains (Owhat) & RM-01 & FMM & 27 & 244 & 251 & 47.6/1.9 (mm/in) - N core & NX & TD \\
\hline Russian Mountains (Owhat) & RM-02 & FMM & 1 & 5 & 14 & $47.6 / 1.9(\mathrm{~mm} / \mathrm{in})$ - N core & NX & \\
\hline Russian Mountains (Owhat) & RM-02 & FMM & 2 & 14 & 24 & $47.6 / 1.9(\mathrm{~mm} / \mathrm{in})$ - N core & NX & \\
\hline Russian Mountains (Owhat) & RM-02 & FMM & 3 & 24 & 33 & $47.6 / 1.9(\mathrm{~mm} / \mathrm{in})$ - N core & $\mathrm{NX}$ & \\
\hline Russian Mountains (Owhat) & RM-02 & FMM & 4 & 33 & 42 & $47.6 / 1.9(\mathrm{~mm} / \mathrm{in})$ - N core & NX & \\
\hline Russian Mountains (Owhat) & RM-02 & FMM & 5 & 42 & 51.5 & 47.6/1.9 (mm/in) - N core & NX & \\
\hline
\end{tabular}




\section{Alaska GMC Russian Mountains Prospect Inventory}

Russian Mountains (Owhat) Russian Mountains (Owhat) Russian Mountains (Owhat) Russian Mountains (Owhat) Russian Mountains (Owhat) Russian Mountains (Owhat) Russian Mountains (Owhat) Russian Mountains (Owhat) Russian Mountains (Owhat) Russian Mountains (Owhat) Russian Mountains (Owhat) Russian Mountains (Owhat) Russian Mountains (Owhat) Russian Mountains (Owhat) Russian Mountains (Owhat) Russian Mountains (Owhat) Russian Mountains (Owhat) Russian Mountains (Owhat) Russian Mountains (Owhat) Russian Mountains (Owhat) Russian Mountains (Owhat) Russian Mountains (Owhat) Russian Mountains (Owhat) Russian Mountains (Owhat) Russian Mountains (Owhat) Russian Mountains (Owhat) Russian Mountains (Owhat) Russian Mountains (Owhat) Russian Mountains (Owhat) Russian Mountains (Owhat) Russian Mountains (Owhat) Russian Mountains (Owhat) Russian Mountains (Owhat)

\begin{tabular}{ll|l} 
RM-02 & FMM \\
RM-02 & FMM \\
RM-02 & FMM \\
RM-02 & FMM \\
RM-02 & FMM \\
RM-02 & FMM \\
RM-02 & FMM \\
RM-02 & FMM \\
RM-02 & FMM \\
RM-02 & FMM \\
RM-02 & FMM \\
RM-02 & FMM \\
RM-02 & FMM \\
RM-02 & FMM \\
RM-02 & FMM \\
RM-03 & FMM \\
RM-03 & FMM \\
RM-03 & FMM \\
RM-03 & FMM \\
RM-03 & FMM \\
RM-03 & FMM \\
RM-03 & FMM \\
RM-03 & FMM \\
RM-03 & FMM \\
RM-03 & FMM \\
RM-03 & FMM \\
RM-03 & FMM \\
RM-03 & FMM \\
RM-03 & FMM \\
RM-03 & FMM \\
RM-03 & FMM \\
RM-03 & FMM \\
RM-03 & FMM \\
\end{tabular}

\begin{tabular}{|c|c|c|c|c|c|}
\hline 6 & 51.5 & 61 & 47.6/1.9 (mm/in) - N core & NX & \\
\hline 7 & 61 & 70 & $47.6 / 1.9(\mathrm{~mm} / \mathrm{in})-\mathrm{N}$ core & $N X$ & \\
\hline 8 & 70 & 80 & 47.6/1.9 (mm/in) - N core & NX & \\
\hline 9 & 80 & 90 & $47.6 / 1.9$ (mm/in) - $\mathrm{N}$ core & $N X$ & \\
\hline 10 & 90 & 99 & $47.6 / 1.9(\mathrm{~mm} / \mathrm{in})$ - $\mathrm{N}$ core & $N X$ & \\
\hline 11 & 99 & 109 & $47.6 / 1.9(\mathrm{~mm} / \mathrm{in})-\mathrm{N}$ core & $N X$ & \\
\hline 12 & 109 & 118 & 47.6/1.9 (mm/in) - $\mathrm{N}$ core & $N X$ & \\
\hline 13 & 118 & 128 & $47.6 / 1.9(\mathrm{~mm} / \mathrm{in})$ - $\mathrm{N}$ core & $N X$ & \\
\hline 14 & 128 & 138 & $47.6 / 1.9$ (mm/in) - $\mathrm{N}$ core & $N X$ & \\
\hline 15 & 138 & 147 & $47.6 / 1.9(\mathrm{~mm} / \mathrm{in})-\mathrm{N}$ core & NX & \\
\hline 16 & 147 & 156.5 & $47.6 / 1.9(\mathrm{~mm} / \mathrm{in})$ - $\mathrm{N}$ core & NX & \\
\hline 17 & 156.5 & 166 & $47.6 / 1.9(\mathrm{~mm} / \mathrm{in})-\mathrm{N}$ core & NX & \\
\hline 18 & 166 & & 47.6/1.9 (mm/in) - $\mathrm{N}$ core & NX & missing box \\
\hline 19 & & 177.5 & $47.6 / 1.9(\mathrm{~mm} / \mathrm{in})$ - $\mathrm{N}$ core & NX & missing box \\
\hline 20 & 177.5 & 187 & $47.6 / 1.9(\mathrm{~mm} / \mathrm{in})-\mathrm{N}$ core & NX & TD \\
\hline 1 & 0 & 19.5 & $47.6 / 1.9(\mathrm{~mm} / \mathrm{in})$ - $\mathrm{N}$ core & $\mathrm{NX}$ & jumbled core \\
\hline 2 & 19.5 & 31 & $47.6 / 1.9(\mathrm{~mm} / \mathrm{in})-\mathrm{N}$ core & NX & \\
\hline 3 & 31 & 43 & $47.6 / 1.9(\mathrm{~mm} / \mathrm{in})-\mathrm{N}$ core & $\mathrm{NX}$ & \\
\hline 4 & 43 & 54 & $47.6 / 1.9(\mathrm{~mm} / \mathrm{in})$ - $\mathrm{N}$ core & NX & \\
\hline 5 & 54 & 64 & 47.6/1.9 (mm/in) - $\mathrm{N}$ core & NX & dumped and jumbled core \\
\hline 6 & 64 & 74 & $47.6 / 1.9(\mathrm{~mm} / \mathrm{in})$ - $\mathrm{N}$ core & NX & \\
\hline 7 & 74 & 83.5 & $47.6 / 1.9(\mathrm{~mm} / \mathrm{in})-\mathrm{N}$ core & NX & \\
\hline 8 & 83.5 & 93 & $47.6 / 1.9(\mathrm{~mm} / \mathrm{in})-\mathrm{N}$ core & NX & \\
\hline 9 & 93 & 102.5 & 47.6/1.9 (mm/in) - $\mathrm{N}$ core & $N X$ & \\
\hline 10 & 102.5 & 112 & $47.6 / 1.9(\mathrm{~mm} / \mathrm{in})-\mathrm{N}$ core & $N X$ & \\
\hline 11 & 111 & 121 & $47.6 / 1.9(\mathrm{~mm} / \mathrm{in})$ - $\mathrm{N}$ core & $N X$ & \\
\hline 12 & 121 & 131 & $47.6 / 1.9(\mathrm{~mm} / \mathrm{in})-\mathrm{N}$ core & NX & \\
\hline 13 & 131 & 141 & $47.6 / 1.9(\mathrm{~mm} / \mathrm{in})-\mathrm{N}$ core & $N X$ & \\
\hline 14 & 141 & 150.5 & $47.6 / 1.9(\mathrm{~mm} / \mathrm{in})-\mathrm{N}$ core & $N X$ & \\
\hline 15 & 150.5 & 160 & $47.6 / 1.9(\mathrm{~mm} / \mathrm{in})$ - $\mathrm{N}$ core & NX & \\
\hline 16 & 160 & 168 & $47.6 / 1.9(\mathrm{~mm} / \mathrm{in})-\mathrm{N}$ core & NX & \\
\hline 17 & 168 & 177 & $47.6 / 1.9(\mathrm{~mm} / \mathrm{in})-\mathrm{N}$ core & NX & dumped and lost=roadkill \\
\hline 18 & 177 & 186 & 47.6/1.9 (mm/in) - $\mathrm{N}$ core & $N X$ & \\
\hline
\end{tabular}




\section{Alaska GMC Russian Mountains Prospect Inventory}

Russian Mountains (Owhat) Russian Mountains (Owhat) Russian Mountains (Owhat) Russian Mountains (Owhat) Russian Mountains (Owhat) Russian Mountains (Owhat) Russian Mountains (Owhat) Russian Mountains (Owhat) Russian Mountains (Owhat) Russian Mountains (Owhat) Russian Mountains (Owhat) Russian Mountains (Owhat) Russian Mountains (Owhat) Russian Mountains (Owhat) Russian Mountains (Owhat) Russian Mountains (Owhat) Russian Mountains (Owhat) Russian Mountains (Owhat) Russian Mountains (Owhat) Russian Mountains (Owhat) Russian Mountains (Owhat) Russian Mountains (Owhat) Russian Mountains (Owhat) Russian Mountains (Owhat) Russian Mountains (Owhat) Russian Mountains (Owhat) Russian Mountains (Owhat) Russian Mountains (Owhat) Russian Mountains (Owhat) Russian Mountains (Owhat) Russian Mountains (Owhat) Russian Mountains (Owhat) Russian Mountains (Owhat)

\begin{tabular}{|l|l} 
RM-03 & FMM \\
RM-03 & FMM \\
RM-03 & FMM \\
RM-03 & FMM \\
RM-03 & FMM \\
RM-03 & FMM \\
RM-03 & FMM \\
RM-03 & FMM \\
RM-03 & FMM \\
RM-03 & FMM \\
RM-03 & FMM \\
RM-03 & FMM \\
RM-03 & FMM \\
RM-03 & FMM \\
RM-03 & FMM \\
RM-03 & FMM \\
RM-03 & FMM \\
RM-03 & FMM \\
RM-03 & FMM \\
RM-03 & FMM \\
RM-03 & FMM \\
RM-03 & FMM \\
RM-03 & FMM \\
RM-04 & FMM \\
RM-04 & FMM \\
RM-04 & FMM \\
RM-04 & FMM \\
RM-04 & FMM \\
RM-04 & FMM \\
RM-04 & FMM \\
RM-04 & FMM \\
RM-04 & FMM \\
RM-04 & FMM \\
&
\end{tabular}

\begin{tabular}{|c|c|c|c|c|c|}
\hline 19 & 186 & 195.5 & $47.6 / 1.9(\mathrm{~mm} / \mathrm{in})-\mathrm{N}$ core & NX & \\
\hline 20 & 195.5 & 205 & $47.6 / 1.9(\mathrm{~mm} / \mathrm{in})-\mathrm{N}$ core & $N X$ & jumbled core \\
\hline 21 & 205 & 215 & $47.6 / 1.9(\mathrm{~mm} / \mathrm{in})$ - $\mathrm{N}$ core & NX & \\
\hline 22 & 215 & 224 & $47.6 / 1.9(\mathrm{~mm} / \mathrm{in})$ - $\mathrm{N}$ core & $N X$ & \\
\hline 23 & 224 & 233 & 47.6/1.9 (mm/in) - N core & $\mathrm{NX}$ & \\
\hline 24 & 233 & 243 & $47.6 / 1.9(\mathrm{~mm} / \mathrm{in})$ - $\mathrm{N}$ core & $\mathrm{NX}$ & \\
\hline 25 & 243 & 252.5 & 47.6/1.9 (mm/in) - $\mathrm{N}$ core & $\mathrm{NX}$ & \\
\hline 26 & 252.5 & 262.5 & $47.6 / 1.9(\mathrm{~mm} / \mathrm{in})$ - $\mathrm{N}$ core & $N X$ & \\
\hline 27 & 262.5 & 272.5 & $47.6 / 1.9(\mathrm{~mm} / \mathrm{in})$ - $\mathrm{N}$ core & $N X$ & \\
\hline 28 & 272.5 & 281.5 & $47.6 / 1.9(\mathrm{~mm} / \mathrm{in})$ - $\mathrm{N}$ core & $\mathrm{NX}$ & \\
\hline 29 & 281.5 & 292.5 & $47.6 / 1.9(\mathrm{~mm} / \mathrm{in})$ - $\mathrm{N}$ core & $N X$ & \\
\hline 30 & 292.5 & 302.5 & 47.6/1.9 (mm/in) - N core & $\mathrm{NX}$ & \\
\hline 31 & 302.5 & 311.5 & $47.6 / 1.9(\mathrm{~mm} / \mathrm{in})-\mathrm{N}$ core & $\mathrm{NX}$ & \\
\hline 32 & 311.5 & 321 & $47.6 / 1.9(\mathrm{~mm} / \mathrm{in})$ - $\mathrm{N}$ core & $N X$ & dumped and lost=roadkill \\
\hline 33 & 321 & 330.5 & 47.6/1.9 (mm/in) - N core & $\mathrm{NX}$ & jumbled core \\
\hline 34 & 330.5 & 340.5 & $47.6 / 1.9(\mathrm{~mm} / \mathrm{in})$ - $\mathrm{N}$ core & $\mathrm{NX}$ & \\
\hline 35 & 340.5 & 350 & $47.6 / 1.9(\mathrm{~mm} / \mathrm{in})$ - $\mathrm{N}$ core & $\mathrm{NX}$ & \\
\hline 36 & 350 & 359 & $47.6 / 1.9(\mathrm{~mm} / \mathrm{in})$ - $\mathrm{N}$ core & $N X$ & \\
\hline 37 & 359 & 369 & $47.6 / 1.9(\mathrm{~mm} / \mathrm{in})$ - $\mathrm{N}$ core & $\mathrm{NX}$ & missing box \\
\hline 38 & 369 & 378.5 & 47.6/1.9 (mm/in) - N core & $\mathrm{NX}$ & \\
\hline 39 & 378.5 & 387.5 & $47.6 / 1.9(\mathrm{~mm} / \mathrm{in})$ - $\mathrm{N}$ core & $N X$ & \\
\hline 40 & 387.5 & 397.5 & 47.6/1.9 (mm/in) - N core & $N X$ & \\
\hline 41 & 397.5 & 401 & $47.6 / 1.9(\mathrm{~mm} / \mathrm{in})$ - $\mathrm{N}$ core & NX & TD \\
\hline 1 & 10 & 36 & $47.6 / 1.9(\mathrm{~mm} / \mathrm{in})$ - N core & $\mathrm{NX}$ & no box lid \\
\hline 2 & 36 & 48.5 & $47.6 / 1.9(\mathrm{~mm} / \mathrm{in})-\mathrm{N}$ core & $N X$ & \\
\hline 3 & 48.5 & 58.5 & $47.6 / 1.9(\mathrm{~mm} / \mathrm{in})$ - $\mathrm{N}$ core & NX & \\
\hline 4 & 58.5 & 68.5 & $47.6 / 1.9(\mathrm{~mm} / \mathrm{in})$ - $\mathrm{N}$ core & $\mathrm{NX}$ & \\
\hline 5 & 70.5 & 80 & $47.6 / 1.9(\mathrm{~mm} / \mathrm{in})$ - $\mathrm{N}$ core & $N X$ & \\
\hline 6 & 80 & 89 & 47.6/1.9 (mm/in) - $\mathrm{N}$ core & $\mathrm{NX}$ & \\
\hline 7 & 89 & 98.5 & $47.6 / 1.9(\mathrm{~mm} / \mathrm{in})$ - $\mathrm{N}$ core & $N X$ & \\
\hline 8 & 98.5 & 108.5 & $47.6 / 1.9(\mathrm{~mm} / \mathrm{in})$ - $\mathrm{N}$ core & $N X$ & \\
\hline 9 & 108.5 & 116.5 & $47.6 / 1.9(\mathrm{~mm} / \mathrm{in})$ - $\mathrm{N}$ core & $\mathrm{NX}$ & \\
\hline 10 & 116.5 & 126 & $47.6 / 1.9(\mathrm{~mm} / \mathrm{in})$ - $\mathrm{N}$ core & $N X$ & \\
\hline
\end{tabular}




\section{Alaska GMC Russian Mountains Prospect Inventory}

Russian Mountains (Owhat) Russian Mountains (Owhat) Russian Mountains (Owhat) Russian Mountains (Owhat) Russian Mountains (Owhat) Russian Mountains (Owhat) Russian Mountains (Owhat) Russian Mountains (Owhat) Russian Mountains (Owhat) Russian Mountains (Owhat) Russian Mountains (Owhat) Russian Mountains (Owhat) Russian Mountains (Owhat) Russian Mountains (Owhat) Russian Mountains (Louise) Russian Mountains (Louise) Russian Mountains (Louise) Russian Mountains (Louise) Russian Mountains (Louise) Russian Mountains (Louise) Russian Mountains (Louise) Russian Mountains (Louise) Russian Mountains (Louise) Russian Mountains (Louise) Russian Mountains (Louise) Russian Mountains (Louise) Russian Mountains (Louise) Russian Mountains (Louise) Russian Mountains (Louise) Russian Mountains (Louise) Russian Mountains (Louise) Russian Mountains (Louise) Russian Mountains (Louise)

\begin{tabular}{|l|l} 
RM-04 & FMM \\
RM-04 & FMM \\
RM-04 & FMM \\
RM-04 & FMM \\
RM-04 & FMM \\
RM-04 & FMM \\
RM-04 & FMM \\
RM-04 & FMM \\
RM-04 & FMM \\
RM-04 & FMM \\
RM-04 & FMM \\
RM-04 & FMM \\
RM-04 & FMM \\
RM-04 & FMM \\
RM-05 & FMM \\
RM-05 & FMM \\
RM-05 & FMM \\
RM-05 & FMM \\
RM-05 & FMM \\
RM-05 & FMM \\
RM-05 & FMM \\
RM-05 & FMM \\
RM-05 & FMM \\
RM-05 & FMM \\
RM-05 & FMM \\
RM-05 & FMM \\
RM-05 & FMM \\
RM-05 & FMM \\
RM-05 & FMM \\
RM-05 & FMM \\
RM-05 & FMM \\
RM-05 & FMM \\
RM-05 & FMM \\
\end{tabular}

\begin{tabular}{|c|c|c|c|c|c|}
\hline 11 & 126 & 136 & 47.6/1.9 (mm/in) - N core & NX & \\
\hline 12 & 136 & 146 & $47.6 / 1.9(\mathrm{~mm} / \mathrm{in})$ - $\mathrm{N}$ core & $N X$ & \\
\hline 13 & 146 & 155 & 47.6/1.9 (mm/in) - N core & NX & \\
\hline 14 & 155 & 164.5 & 47.6/1.9 (mm/in) - N core & $\mathrm{NX}$ & \\
\hline 15 & 164.5 & 174.5 & 47.6/1.9 (mm/in) - N core & $\mathrm{NX}$ & \\
\hline 16 & 174.5 & 184 & 47.6/1.9 (mm/in) - N core & $\mathrm{NX}$ & \\
\hline 17 & 184 & 193 & 47.6/1.9 (mm/in) - N core & $\mathrm{NX}$ & \\
\hline 18 & 193 & 202.5 & 47.6/1.9 (mm/in) - N core & $N X$ & \\
\hline 19 & 202.5 & 212 & 47.6/1.9 (mm/in) - N core & $\mathrm{NX}$ & \\
\hline 20 & 212 & 221 & 47.6/1.9 (mm/in) - N core & $\mathrm{NX}$ & \\
\hline 21 & 221 & 230 & 47.6/1.9 (mm/in) - N core & $N X$ & \\
\hline 22 & 230 & 240 & 47.6/1.9 (mm/in) - N core & $\mathrm{NX}$ & \\
\hline 23 & 240 & 250 & 47.6/1.9 (mm/in) - N core & NX & \\
\hline 24 & 250 & 250.5 & 47.6/1.9 (mm/in) - N core & $N X$ & TD \\
\hline 1 & 20 & 30 & 47.6/1.9 (mm/in) - N core & NX & \\
\hline 2 & 30 & 39.5 & 47.6/1.9 (mm/in) - N core & $\mathrm{NX}$ & \\
\hline 3 & 39.5 & 49 & 47.6/1.9 (mm/in) - N core & $\mathrm{NX}$ & \\
\hline 4 & 49 & 59 & 47.6/1.9 (mm/in) - N core & $\mathrm{NX}$ & \\
\hline 5 & 59 & 68.5 & 47.6/1.9 (mm/in) - N core & $\mathrm{NX}$ & \\
\hline 6 & 68.5 & 78 & 47.6/1.9 (mm/in) - N core & $\mathrm{NX}$ & \\
\hline 7 & 78 & 89.5 & 47.6/1.9 (mm/in) - N core & $N X$ & \\
\hline 8 & 89.5 & 97 & 47.6/1.9 (mm/in) - N core & $N X$ & \\
\hline 9 & 97 & 106 & 47.6/1.9 (mm/in) - N core & $N X$ & \\
\hline 10 & 106 & 117 & 47.6/1.9 (mm/in) - N core & NX & \\
\hline 11 & 117 & 126 & 47.6/1.9 (mm/in) - N core & $N X$ & \\
\hline 12 & 126 & 135.5 & 47.6/1.9 (mm/in) - N core & $N X$ & \\
\hline 13 & 135.5 & 144.5 & $47.6 / 1.9(\mathrm{~mm} / \mathrm{in})$ - N core & NX & \\
\hline 14 & 144.5 & 154 & 47.6/1.9 (mm/in) - N core & $N X$ & \\
\hline 15 & 154 & 163 & 47.6/1.9 (mm/in) - N core & $\mathrm{NX}$ & \\
\hline 16 & 163 & 173 & 47.6/1.9 (mm/in) - N core & $N X$ & \\
\hline 17 & 173 & 182.5 & 47.6/1.9 (mm/in) - N core & $\mathrm{NX}$ & \\
\hline 18 & 182.5 & 192 & 47.6/1.9 (mm/in) - N core & $\mathrm{NX}$ & \\
\hline 19[ & 192 & 201.5 & 47.6/1.9 (mm/in) - N core & NX & \\
\hline
\end{tabular}




\section{Alaska GMC Russian Mountains Prospect Inventory}

Russian Mountains (Louise) Russian Mountains (Louise) Russian Mountains (Louise) Russian Mountains (Louise) Russian Mountains (Louise) Russian Mountains (Louise) Russian Mountains (Louise) Russian Mountains (Louise) Russian Mountains (Louise) Russian Mountains (Louise) Russian Mountains (Louise) Russian Mountains (Louise) Russian Mountains (Louise) Russian Mountains (Louise) Russian Mountains (Louise) Russian Mountains (Louise) Russian Mountains (Louise) Russian Mountains (Louise) Russian Mountains (Louise) Russian Mountains (Louise) Russian Mountains (Louise) Russian Mountains (Louise) Russian Mountains (Louise) Russian Mountains (Louise) Russian Mountains (Louise) Russian Mountains (Louise) Russian Mountains (Louise) Russian Mountains (Headwall) Russian Mountains (Headwall) Russian Mountains (Headwall) Russian Mountains (Headwall) Russian Mountains (Headwall) Russian Mountains (Headwall)

\begin{tabular}{|l|l} 
RM-05 & FMM \\
RM-05 & FMM \\
RM-05 & FMM \\
RM-05 & FMM \\
RM-05 & FMM \\
RM-05 & FMM \\
RM-05 & FMM \\
RM-05 & FMM \\
RM-05 & FMM \\
RM-05 & FMM \\
RM-05 & FMM \\
RM-05 & FMM \\
RM-05 & FMM \\
RM-05 & FMM \\
RM-05 & FMM \\
RM-05 & FMM \\
RM-05 & FMM \\
RM-05 & FMM \\
RM-05 & FMM \\
RM-05 & FMM \\
RM-05 & FMM \\
RM-05 & FMM \\
RM-05 & FMM \\
RM-05 & FMM \\
RM-05 & FMM \\
RM-05 & FMM \\
RM-05 & FMM \\
RM-06 & FMM \\
RM-06 & FMM \\
RM-06 & FMM \\
RM-06 & FMM \\
RM-06 & FMM \\
RM-06 & FMM \\
\end{tabular}

\begin{tabular}{|c|c|c|c|c|c|}
\hline 20 & 201.5 & 211 & $47.6 / 1.9(\mathrm{~mm} / \mathrm{in})$ - N core & $\mathrm{NX}$ & \\
\hline 21 & 211 & 220.5 & $47.6 / 1.9(\mathrm{~mm} / \mathrm{in})$ - N core & $N X$ & \\
\hline 22 & 220.5 & 230 & $47.6 / 1.9(\mathrm{~mm} / \mathrm{in})$ - N core & $\mathrm{NX}$ & \\
\hline 23 & 230 & 240 & 47.6/1.9 (mm/in) - N core & NX & \\
\hline 24 & 240 & 249 & 47.6/1.9 (mm/in) - N core & $\mathrm{NX}$ & \\
\hline 25 & 249 & 258 & $47.6 / 1.9(\mathrm{~mm} / \mathrm{in})$ - $\mathrm{N}$ core & $\mathrm{NX}$ & \\
\hline 26 & 258 & 267.5 & $47.6 / 1.9(\mathrm{~mm} / \mathrm{in})$ - N core & NX & \\
\hline 27 & 267.5 & 277 & $47.6 / 1.9(\mathrm{~mm} / \mathrm{in})$ - N core & $\mathrm{NX}$ & \\
\hline 28 & 277 & 286.5 & $47.6 / 1.9(\mathrm{~mm} / \mathrm{in})$ - N core & $\mathrm{NX}$ & \\
\hline 29 & 286.5 & 296.5 & $47.6 / 1.9(\mathrm{~mm} / \mathrm{in})$ - N core & $\mathrm{NX}$ & \\
\hline 30 & 296.5 & 306.5 & $47.6 / 1.9(\mathrm{~mm} / \mathrm{in})$ - N core & $\mathrm{NX}$ & \\
\hline 31 & 306.5 & 315.5 & 47.6/1.9 (mm/in) - N core & NX & \\
\hline 32 & 315.5 & 325.5 & $47.6 / 1.9(\mathrm{~mm} / \mathrm{in})$ - N core & $\mathrm{NX}$ & \\
\hline 33 & 325.5 & 335 & 47.6/1.9 (mm/in) - N core & $\mathrm{NX}$ & \\
\hline 34 & 335 & 344.5 & $47.6 / 1.9(\mathrm{~mm} / \mathrm{in})$ - N core & NX & \\
\hline 35 & 344.5 & 354 & 47.6/1.9 (mm/in) - N core & $\mathrm{NX}$ & \\
\hline 36 & 354 & 363.5 & 47.6/1.9 (mm/in) - N core & $\mathrm{NX}$ & \\
\hline 37 & 363.5 & 372.5 & $47.6 / 1.9(\mathrm{~mm} / \mathrm{in})$ - N core & NX & \\
\hline 38 & 372.5 & 382 & $47.6 / 1.9(\mathrm{~mm} / \mathrm{in})$ - N core & $\mathrm{NX}$ & \\
\hline 39 & 382 & 391.5 & 47.6/1.9 (mm/in) - N core & NX & \\
\hline 40 & 391.5 & 401 & $47.6 / 1.9(\mathrm{~mm} / \mathrm{in})$ - N core & $\mathrm{NX}$ & \\
\hline 41 & 401 & 410.5 & 47.6/1.9 (mm/in) - N core & $\mathrm{NX}$ & \\
\hline 42 & 410.5 & 420 & 47.6/1.9 (mm/in) - N core & $\mathrm{NX}$ & \\
\hline 43 & 420 & 429.5 & 47.6/1.9 (mm/in) - N core & $\mathrm{NX}$ & \\
\hline 44 & 429.5 & 439 & $47.6 / 1.9(\mathrm{~mm} / \mathrm{in})$ - N core & $\mathrm{NX}$ & \\
\hline 45 & 439 & 448.5 & 47.6/1.9 (mm/in) - N core & NX & \\
\hline 46 & 448.5 & 455 & $47.6 / 1.9(\mathrm{~mm} / \mathrm{in})$ - N core & $\mathrm{NX}$ & TD \\
\hline 1 & 0 & 18 & 47.6/1.9 (mm/in) - N core & $\mathrm{NX}$ & \\
\hline 2 & 18 & 29 & 47.6/1.9 (mm/in) - N core & $\mathrm{NX}$ & \\
\hline 3 & 29 & 42.5 & $47.6 / 1.9(\mathrm{~mm} / \mathrm{in})$ - N core & $\mathrm{NX}$ & \\
\hline 4 & 42.5 & 53.5 & $47.6 / 1.9(\mathrm{~mm} / \mathrm{in})$ - $\mathrm{N}$ core & $\mathrm{NX}$ & \\
\hline 5 & 53.5 & 65 & $47.6 / 1.9(\mathrm{~mm} / \mathrm{in})$ - N core & $\mathrm{NX}$ & \\
\hline 6 & 65 & 74.5 & 47.6/1.9 (mm/in) - N core & $\mathrm{NX}$ & \\
\hline
\end{tabular}




\section{Alaska GMC Russian Mountains Prospect Inventory}

\begin{tabular}{l|l} 
Russian Mountains (Headwall) & RM-06
\end{tabular} Russian Mountains (Headwall) RM-06 Russian Mountains (Headwall) RM-06 Russian Mountains (Headwall) RM-06 Russian Mountains (Headwall) RM-06 Russian Mountains (Headwall) RM-06 Russian Mountains (Headwall) RM-06 Russian Mountains (Headwall) RM-06 Russian Mountains (Headwall) RM-06 Russian Mountains (Headwall) RM-06 Russian Mountains (Headwall) RM-06 Russian Mountains (Headwall) RM-06 Russian Mountains (Headwall) RM-06 Russian Mountains (Headwall) RM-06 Russian Mountains (Headwall) RM-06 Russian Mountains (Headwall) RM-06 Russian Mountains (Headwall) RM-06 Russian Mountains (Headwall) RM-06 Russian Mountains (Headwall) RM-06 Russian Mountains (Headwall) RM-06 Russian Mountains (Headwall) RM-06 Russian Mountains (Headwall) RM-06 Russian Mountains (Headwall) RM-06 Russian Mountains (Headwall) RM-06 Russian Mountains (Headwall) RM-06 Russian Mountains (Headwall) RM-06 Russian Mountains (Headwall) RM-06 Russian Mountains (Headwall) RM-06 Russian Mountains (Headwall) RM-06 Russian Mountains (Headwall) RM-06 Russian Mountains (Headwall) RM-06 Russian Mountains (Headwall) RM-06 Russian Mountains (Headwall) RM-06

FMM
FMM
FMM
FMM
FMM
FMM
FMM
FMM
FMM
FMM
FMM
FMM
FMM
FMM
FMM
FMM
FMM
FMM
FMM
FMM
FMM
FMM
FMM
FMM
FMM
FMM
FMM
FMM
FMM
FMM
FMM
FMM
FMM

\begin{tabular}{|c|c|c|c|c|c|}
\hline 7 & 74.5 & 83.5 & 47.6/1.9 (mm/in) - N core & NX & \\
\hline 8 & 83.5 & 92.5 & 47.6/1.9 (mm/in) - N core & NX & \\
\hline 9 & 92.5 & 102.5 & 47.6/1.9 (mm/in) - N core & NX & \\
\hline 10 & 102.5 & 111 & 47.6/1.9 (mm/in) - N core & NX & \\
\hline 11 & 111 & 120.5 & 47.6/1.9 (mm/in) - N core & NX & \\
\hline 12 & 120.5 & 130 & 47.6/1.9 (mm/in) - N core & NX & \\
\hline 13 & 130 & 139 & 47.6/1.9 (mm/in) - $\mathrm{N}$ core & NX & \\
\hline 14 & 139 & 148.5 & 47.6/1.9 (mm/in) - N core & NX & \\
\hline 15 & 148.5 & 158 & 47.6/1.9 (mm/in) - N core & NX & \\
\hline 16 & 158 & 167.5 & 47.6/1.9 (mm/in) - N core & NX & \\
\hline 17 & 167.5 & 176.5 & 47.6/1.9 (mm/in) - N core & NX & \\
\hline 18 & 176.5 & 186.5 & 47.6/1.9 (mm/in) - N core & NX & \\
\hline 19 & 186.5 & 196 & 47.6/1.9 (mm/in) - N core & NX & \\
\hline 20 & 196 & 205.5 & 47.6/1.9 (mm/in) - N core & NX & \\
\hline 21 & 205.5 & 215 & 47.6/1.9 (mm/in) - N core & NX & \\
\hline 22 & 215 & 224.5 & 47.6/1.9 (mm/in) - N core & NX & \\
\hline 23 & 224.5 & 234 & 47.6/1.9 (mm/in) - N core & NX & \\
\hline 24 & 234 & 243.5 & 47.6/1.9 (mm/in) - $\mathrm{N}$ core & NX & \\
\hline 25 & 243.5 & 253 & 47.6/1.9 (mm/in) - N core & NX & \\
\hline 26 & 253 & 262 & 47.6/1.9 (mm/in) - N core & NX & \\
\hline 27 & 262 & 272 & 47.6/1.9 (mm/in) - N core & NX & \\
\hline 28 & 272 & 282 & 47.6/1.9 (mm/in) - N core & NX & \\
\hline 29 & 282 & 291 & 47.6/1.9 (mm/in) - N core & NX & \\
\hline 30 & 291 & 301.5 & 47.6/1.9 (mm/in) - N core & NX & \\
\hline 31 & 301.5 & 310 & 47.6/1.9 (mm/in) - N core & NX & \\
\hline 32 & 310 & 320 & 47.6/1.9 (mm/in) - N core & NX & \\
\hline 33 & 320 & 330 & 47.6/1.9 (mm/in) - N core & NX & \\
\hline 34 & 330 & 339 & 47.6/1.9 (mm/in) - N core & NX & \\
\hline 35 & 339 & 349 & 47.6/1.9 (mm/in) - $\mathrm{N}$ core & NX & \\
\hline 36 & 349 & 358 & 47.6/1.9 (mm/in) - N core & NX & \\
\hline 37 & 358 & 368 & 47.6/1.9 (mm/in) - N core & NX & \\
\hline 38 & 368 & 377.5 & 47.6/1.9 (mm/in) - N core & NX & \\
\hline 39 & 377.5 & 387 & 47.6/1.9 (mm/in) - N core & NX & \\
\hline
\end{tabular}




\section{Alaska GMC Russian Mountains Prospect Inventory}

\begin{tabular}{l|l} 
Russian Mountains (Headwall) & RM-06
\end{tabular} Russian Mountains (Headwall) RM-06 Russian Mountains (Headwall) RM-06 Russian Mountains (Headwall) RM-06 Russian Mountains (Headwall) RM-06 Russian Mountains (Headwall) RM-06 Russian Mountains (Headwall) RM-06 Russian Mountains (Headwall) RM-06 Russian Mountains (Headwall) RM-06 Russian Mountains (Headwall) RM-06 Russian Mountains (Headwall) RM-06 Russian Mountains (Headwall) RM-06 Russian Mountains (Headwall) RM-06 Russian Mountains (Headwall) RM-06 Russian Mountains (Headwall) RM-06 Russian Mountains (Headwall) RM-06 Russian Mountains (Headwall) RM-06 Russian Mountains (Headwall) RM-06 Russian Mountains (Headwall) RM-06 Russian Mountains (Headwall) RM-06 Russian Mountains (Headwall) RM-06 Russian Mountains (Headwall) RM-07 Russian Mountains (Headwall) RM-07 Russian Mountains (Headwall) RM-07 Russian Mountains (Headwall) RM-07 Russian Mountains (Headwall) RM-07 Russian Mountains (Headwall) RM-07 Russian Mountains (Headwall) RM-07 Russian Mountains (Headwall) RM-07 Russian Mountains (Headwall) RM-07 Russian Mountains (Headwall) RM-07 Russian Mountains (Headwall) RM-07 \begin{tabular}{l|l} 
Russian Mountains (Headwall) & RM-07
\end{tabular}

FMM
FMM
FMM
FMM
FMM
FMM
FMM
FMM
FMM
FMM
FMM
FMM
FMM
FMM
FMM
FMM
FMM
FMM
FMM
FMM
FMM
FMM
FMM
FMM
FMM
FMM
FMM
FMM
FMM
FMM
FMM
FMM
FMM

\begin{tabular}{|c|c|c|c|c|c|}
\hline 40 & 387 & 396.5 & 47.6/1.9 (mm/in) - N core & NX & \\
\hline 41 & 396.5 & 406 & 47.6/1.9 (mm/in) - N core & NX & \\
\hline 42 & 406 & 416 & 47.6/1.9 (mm/in) - N core & NX & \\
\hline 43 & 416 & 425.5 & 47.6/1.9 (mm/in) - N core & $\mathrm{NX}$ & \\
\hline 44 & 425.5 & 435 & 47.6/1.9 (mm/in) - N core & $N X$ & \\
\hline 45 & 435 & 445 & $47.6 / 1.9(\mathrm{~mm} / \mathrm{in})$ - N core & $\mathrm{NX}$ & \\
\hline 46 & 445 & 454.5 & 47.6/1.9 (mm/in) - N core & $N X$ & \\
\hline 47 & 454.5 & 464.5 & $47.6 / 1.9(\mathrm{~mm} / \mathrm{in})$ - N core & $\mathrm{NX}$ & \\
\hline 48 & 464.5 & 474 & 47.6/1.9 (mm/in) - N core & $N X$ & \\
\hline 49 & 474 & 483.5 & 47.6/1.9 (mm/in) - N core & $\mathrm{NX}$ & \\
\hline 50 & 483.5 & 493 & 47.6/1.9 (mm/in) - N core & $\mathrm{NX}$ & \\
\hline 51 & 493 & 501 & 47.6/1.9 (mm/in) - N core & NX & \\
\hline 52 & 501 & 511 & 47.6/1.9 (mm/in) - N core & $N X$ & \\
\hline 53 & 511 & 520.5 & 47.6/1.9 (mm/in) - N core & $\mathrm{NX}$ & \\
\hline 54 & 520.5 & 530.5 & 47.6/1.9 (mm/in) - $\mathrm{N}$ core & $\mathrm{NX}$ & \\
\hline 55 & 530.5 & 539.5 & 47.6/1.9 (mm/in) - $\mathrm{N}$ core & NX & \\
\hline 56 & 539.5 & 549 & $47.6 / 1.9(\mathrm{~mm} / \mathrm{in})$ - N core & $\mathrm{NX}$ & \\
\hline 57 & 549 & 558.5 & $47.6 / 1.9(\mathrm{~mm} / \mathrm{in})$ - N core & $\mathrm{NX}$ & \\
\hline 58 & 558.5 & 568 & $47.6 / 1.9$ (mm/in) - N core & $\mathrm{NX}$ & \\
\hline 59 & 568 & 577.5 & $47.6 / 1.9(\mathrm{~mm} / \mathrm{in})$ - N core & $\mathrm{NX}$ & \\
\hline 60 & 577.5 & 580 & $47.6 / 1.9(\mathrm{~mm} / \mathrm{in})$ - N core & NX & TD \\
\hline 1 & 0 & 21.5 & 47.6/1.9 (mm/in) - N core & $\mathrm{NX}$ & \\
\hline 2 & 21.5 & 36 & $47.6 / 1.9(\mathrm{~mm} / \mathrm{in})$ - N core & $\mathrm{NX}$ & \\
\hline 3 & 36 & 50 & 47.6/1.9 (mm/in) - N core & $\mathrm{NX}$ & \\
\hline 4 & 50 & 61 & 47.6/1.9 (mm/in) - N core & NX & \\
\hline 5 & 61 & 73 & 47.6/1.9 (mm/in) - $\mathrm{N}$ core & $\mathrm{NX}$ & \\
\hline 6 & 73 & 83 & 47.6/1.9 (mm/in) - $\mathrm{N}$ core & NX & \\
\hline 7 & 83 & 93.5 & 47.6/1.9 (mm/in) - N core & $\mathrm{NX}$ & \\
\hline 8 & 93.5 & 103 & $47.6 / 1.9(\mathrm{~mm} / \mathrm{in})$ - N core & $\mathrm{NX}$ & \\
\hline 9 & 103 & 112.5 & $47.6 / 1.9$ (mm/in) - N core & $\mathrm{NX}$ & \\
\hline 10 & 112.5 & 122 & $47.6 / 1.9$ (mm/in) - N core & $\mathrm{NX}$ & \\
\hline 11 & 122 & 131.5 & $47.6 / 1.9(\mathrm{~mm} / \mathrm{in})$ - N core & $\mathrm{NX}$ & \\
\hline 12 & 131.5 & 141 & 47.6/1.9 (mm/in) - N core & $N X$ & \\
\hline
\end{tabular}




\section{Alaska GMC Russian Mountains Prospect Inventory}

\begin{tabular}{l|l} 
Russian Mountains (Headwall) & RM-07
\end{tabular} Russian Mountains (Headwall) RM-07 Russian Mountains (Headwall) RM-07 Russian Mountains (Headwall) RM-07 Russian Mountains (Headwall) RM-07 Russian Mountains (Headwall) RM-07 Russian Mountains (Headwall) RM-07 Russian Mountains (Headwall) RM-07 Russian Mountains (Headwall) RM-07 Russian Mountains (Headwall) RM-07 Russian Mountains (Headwall) $\mathrm{RM}-07$ Russian Mountains (Headwall) RM-07 Russian Mountains (Headwall) RM-07 Russian Mountains (Headwall) RM-07 Russian Mountains (Headwall) RM-07 Russian Mountains (Headwall) RM-07 Russian Mountains (Headwall) RM-07 Russian Mountains (Headwall) $\mathrm{RM}-07$ Russian Mountains (Headwall) RM-07 Russian Mountains (Headwall) RM-07 Russian Mountains (Headwall) RM-07 Russian Mountains (Headwall) RM-07 Russian Mountains (Headwall) RM-07 Russian Mountains (Headwall) RM-07 Russian Mountains (Headwall) Russian Mountains (Headwall) Russian Mountains (Headwall) Russian Mountains (Headwall) Russian Mountains (Owhat) Russian Mountains (Owhat) Russian Mountains (Owhat) Russian Mountains (Owhat) Russian Mountains (Owhat)

FMM
FMM
FMM
FMM
FMM
FMM
FMM
FMM
FMM
FMM
FMM
FMM
FMM
FMM
FMM
FMM
FMM
FMM
FMM
FMM
FMM
FMM
FMM
FMM
FMM
FMM
FMM
FMM
FMM
FMM
FMM
FMM
FMM

\begin{tabular}{|c|c|c|c|c|c|}
\hline 13 & 141 & 151 & 47.6/1.9 (mm/in) - N core & NX & \\
\hline 14 & 151 & 160.5 & 47.6/1.9 (mm/in) - N core & NX & \\
\hline 15 & 160.5 & 169 & 47.6/1.9 (mm/in) - N core & NX & \\
\hline 16 & 169 & 177.5 & 47.6/1.9 (mm/in) - N core & NX & \\
\hline 17 & 177.5 & 187 & 47.6/1.9 (mm/in) - N core & NX & \\
\hline 18 & 187 & 196.5 & 47.6/1.9 (mm/in) - N core & NX & \\
\hline 19 & 196.5 & 206 & 47.6/1.9 (mm/in) - $\mathrm{N}$ core & NX & \\
\hline 20 & 206 & 215 & 47.6/1.9 (mm/in) - N core & NX & \\
\hline 21 & 215 & 224 & 47.6/1.9 (mm/in) - N core & NX & \\
\hline 22 & 224 & 232.5 & 47.6/1.9 (mm/in) - N core & NX & \\
\hline 23 & 232.5 & 241 & 47.6/1.9 (mm/in) - N core & NX & \\
\hline 24 & 241 & 250 & 47.6/1.9 (mm/in) - N core & NX & \\
\hline 25 & 250 & 259 & 47.6/1.9 (mm/in) - N core & NX & \\
\hline 26 & 259 & 267.5 & 47.6/1.9 (mm/in) - N core & NX & \\
\hline 27 & 267.5 & 277 & 47.6/1.9 (mm/in) - N core & NX & \\
\hline 28 & 277 & 287 & 47.6/1.9 (mm/in) - N core & NX & \\
\hline 29 & 287 & 296 & 47.6/1.9 (mm/in) - N core & NX & \\
\hline 30 & 296 & 306 & 47.6/1.9 (mm/in) - N core & NX & \\
\hline 31 & 306 & 315.5 & 47.6/1.9 (mm/in) - N core & NX & \\
\hline 32 & 315.5 & 325 & 47.6/1.9 (mm/in) - N core & NX & \\
\hline 33 & 325 & 334.5 & 47.6/1.9 (mm/in) - N core & NX & \\
\hline 34 & 334.5 & 344 & 47.6/1.9 (mm/in) - N core & NX & \\
\hline 35 & 344 & 353.5 & 47.6/1.9 (mm/in) - N core & NX & \\
\hline 36 & 353.5 & 363 & 47.6/1.9 (mm/in) - N core & NX & \\
\hline 37 & 363 & 372.5 & 47.6/1.9 (mm/in) - N core & NX & \\
\hline 38 & 372.5 & 382 & 47.6/1.9 (mm/in) - N core & NX & \\
\hline 39 & 382 & 391 & 47.6/1.9 (mm/in) - N core & NX & \\
\hline 40 & 391 & 400.5 & 47.6/1.9 (mm/in) - N core & NX & TD \\
\hline 1 & 0 & 10 & 47.6/1.9 (mm/in) - N core & NX & \\
\hline 2 & 10 & 19.5 & 47.6/1.9 (mm/in) - N core & NX & \\
\hline 3 & 19.5 & 29 & $47.6 / 1.9(\mathrm{~mm} / \mathrm{in})$ - $\mathrm{N}$ core & NX & \\
\hline 4 & 29 & 38.5 & 47.6/1.9 (mm/in) - N core & NX & \\
\hline 5 & 38.5 & 48 & 47.6/1.9 (mm/in) - N core & NX & \\
\hline
\end{tabular}




\section{Alaska GMC Russian Mountains Prospect Inventory}

Russian Mountains (Owhat) Russian Mountains (Owhat) Russian Mountains (Owhat) Russian Mountains (Owhat) Russian Mountains (Owhat) Russian Mountains (Owhat) Russian Mountains (Owhat) Russian Mountains (Owhat) Russian Mountains (Owhat) Russian Mountains (Owhat) Russian Mountains (Owhat) Russian Mountains (Owhat) Russian Mountains (Owhat) Russian Mountains (Owhat) Russian Mountains (Owhat) Russian Mountains (Owhat) Russian Mountains (Owhat) Russian Mountains (Owhat) Russian Mountains (Owhat) Russian Mountains (Owhat) Russian Mountains (Owhat) Russian Mountains (Owhat) Russian Mountains (Owhat) Russian Mountains (Owhat) Russian Mountains (Owhat) Russian Mountains (Owhat) Russian Mountains (Owhat) Russian Mountains (Owhat) Russian Mountains (Owhat) Russian Mountains (Owhat) Russian Mountains (Owhat) Russian Mountains (Owhat) Russian Mountains (Owhat)

\begin{tabular}{|l|l} 
RM-08 & FMM \\
RM-08 & FMM \\
RM-08 & FMM \\
RM-08 & FMM \\
RM-08 & FMM \\
RM-08 & FMM \\
RM-08 & FMM \\
RM-08 & FMM \\
RM-08 & FMM \\
RM-08 & FMM \\
RM-08 & FMM \\
RM-08 & FMM \\
RM-08 & FMM \\
RM-08 & FMM \\
RM-08 & FMM \\
RM-08 & FMM \\
RM-08 & FMM \\
RM-08 & FMM \\
RM-08 & FMM \\
RM-08 & FMM \\
RM-08 & FMM \\
RM-08 & FMM \\
RM-08 & FMM \\
RM-08 & FMM \\
RM-09 & FMM \\
RM-09 & FMM \\
RM-09 & FMM \\
RM-09 & FMM \\
RM-09 & FMM \\
RM-09 & FMM \\
RM-09 & FMM \\
RM-09 & FMM \\
RM-09 & FMM \\
\end{tabular}

\begin{tabular}{|c|c|c|c|c|c|}
\hline 6 & 48 & 57.5 & 47.6/1.9 (mm/in) - N core & NX & \\
\hline 7 & 57.5 & 67 & 47.6/1.9 (mm/in) - N core & NX & \\
\hline 8 & 67 & 77 & 47.6/1.9 (mm/in) - N core & NX & \\
\hline 9 & 77 & 86 & 47.6/1.9 (mm/in) - N core & NX & \\
\hline 10 & 86 & 96 & 47.6/1.9 (mm/in) - N core & NX & \\
\hline 11 & 96 & 105.5 & 47.6/1.9 (mm/in) - N core & NX & \\
\hline 12 & 105.5 & 115.5 & 47.6/1.9 (mm/in) - $\mathrm{N}$ core & NX & \\
\hline 13 & 115.5 & 125 & 47.6/1.9 (mm/in) - N core & NX & \\
\hline 14 & 125 & 134.5 & 47.6/1.9 (mm/in) - N core & NX & \\
\hline 15 & 134.5 & 144 & 47.6/1.9 (mm/in) - N core & NX & \\
\hline 16 & 144 & 153.5 & 47.6/1.9 (mm/in) - N core & NX & \\
\hline 17 & 153.5 & 163 & 47.6/1.9 (mm/in) - N core & NX & \\
\hline 18 & 163 & 172.5 & 47.6/1.9 (mm/in) - N core & NX & \\
\hline 19 & 172.5 & 182 & 47.6/1.9 (mm/in) - N core & NX & \\
\hline 20 & 182 & 191 & 47.6/1.9 (mm/in) - N core & NX & \\
\hline 21 & 191 & 200.5 & 47.6/1.9 (mm/in) - N core & NX & \\
\hline 22 & 200.5 & 210 & 47.6/1.9 (mm/in) - N core & NX & \\
\hline 23 & 210 & 219.5 & 47.6/1.9 (mm/in) - $\mathrm{N}$ core & NX & \\
\hline 24 & 219.5 & 229.5 & 47.6/1.9 (mm/in) - N core & NX & \\
\hline 25 & 229.5 & 239 & 47.6/1.9 (mm/in) - N core & NX & \\
\hline 26 & 239 & 248.5 & 47.6/1.9 (mm/in) - N core & NX & \\
\hline 27 & 248.5 & 258 & 47.6/1.9 (mm/in) - N core & NX & \\
\hline 28 & 258 & 269 & 47.6/1.9 (mm/in) - N core & NX & \\
\hline 29 & 269 & 276 & 47.6/1.9 (mm/in) - N core & NX & TD \\
\hline 1 & 0.3 & 11.5 & 47.6/1.9 (mm/in) - N core & NX & \\
\hline 2 & 11.5 & 21 & 47.6/1.9 (mm/in) - N core & NX & \\
\hline 3 & 21 & 30 & 47.6/1.9 (mm/in) - N core & NX & \\
\hline 4 & 30 & 39 & 47.6/1.9 (mm/in) - N core & NX & \\
\hline 5 & 39 & 48 & 47.6/1.9 (mm/in) - $\mathrm{N}$ core & NX & \\
\hline 6 & 48 & 57.5 & 47.6/1.9 (mm/in) - N core & NX & \\
\hline 7 & 57.5 & 66.5 & 47.6/1.9 (mm/in) - N core & NX & \\
\hline 8 & 66.5 & 75.5 & 47.6/1.9 (mm/in) - N core & NX & \\
\hline 9 & 75.5 & 85 & 47.6/1.9 (mm/in) - N core & NX & \\
\hline
\end{tabular}




\section{Alaska GMC Russian Mountains Prospect Inventory}

Russian Mountains (Owhat) Russian Mountains (Owhat) Russian Mountains (Owhat) Russian Mountains (Owhat) Russian Mountains (Owhat) Russian Mountains (Owhat) Russian Mountains (Owhat) Russian Mountains (Owhat) Russian Mountains (Owhat) Russian Mountains (Owhat) Russian Mountains (Owhat) Russian Mountains (Louise) Russian Mountains (Louise) Russian Mountains (Louise) Russian Mountains (Louise) Russian Mountains (Louise) Russian Mountains (Louise) Russian Mountains (Louise) Russian Mountains (Louise) Russian Mountains (Louise) Russian Mountains (Louise) Russian Mountains (Louise) Russian Mountains (Louise) Russian Mountains (Louise) Russian Mountains (Louise) Russian Mountains (Louise) Russian Mountains (Louise) Russian Mountains (Louise) Russian Mountains (Louise) Russian Mountains (Louise) Russian Mountains (Louise) Russian Mountains (Louise) Russian Mountains (Louise)

\begin{tabular}{|c|c|}
\hline RM-09 & FMM \\
\hline 1-09 & MM \\
\hline १-09 & FMM \\
\hline И-09 & FMM \\
\hline M-09 & FMM \\
\hline M-09 & FMM \\
\hline И-09 & FMM \\
\hline M-09 & FMM \\
\hline И-09 & FMM \\
\hline RM-09 & FMM \\
\hline M-09 & FMM \\
\hline M-010 & FMM \\
\hline RM-010 & FMM \\
\hline M-O & FMM \\
\hline RM-010 & FMM \\
\hline RM-010 & FMM \\
\hline $\mathrm{R} M-010$ & FMM \\
\hline $\mathrm{M}-010$ & FMM \\
\hline RM-010 & FMN \\
\hline avr & FMM \\
\hline RM-010 & FMM \\
\hline RM-010 & FMM \\
\hline RM-010 & FMN \\
\hline RM-010 & FMM \\
\hline$\sqrt{1}-011$ & FMM \\
\hline RM-011 & FMM \\
\hline RM-011 & FMM \\
\hline RM-011 & FMM \\
\hline RM-011 & FMM \\
\hline RM-0 & FMN \\
\hline RM-011 & FMM \\
\hline RM-011 & FMN \\
\hline |VI-OI? & FMM \\
\hline
\end{tabular}

\begin{tabular}{|c|c|c|c|c|c|}
\hline 10 & 85 & 94 & 47.6/1.9 (mm/in) - N core & NX & \\
\hline 11 & 94 & 103 & 47.6/1.9 (mm/in) - N core & NX & \\
\hline 12 & 103 & 112 & 47.6/1.9 (mm/in) - N core & NX & \\
\hline 13 & 112 & 120.5 & 47.6/1.9 (mm/in) - N core & NX & \\
\hline 14 & 120.5 & 130 & 47.6/1.9 (mm/in) - N core & NX & \\
\hline 15 & 130 & 139.5 & 47.6/1.9 (mm/in) - N core & NX & \\
\hline 16 & 139.5 & 149 & $47.6 / 1.9(\mathrm{~mm} / \mathrm{in})-\mathrm{N}$ core & NX & \\
\hline 17 & 149 & 158 & 47.6/1.9 (mm/in) - N core & NX & \\
\hline 18 & 158 & 166 & 47.6/1.9 (mm/in) - N core & NX & \\
\hline 19 & 166 & 175.5 & $47.6 / 1.9(\mathrm{~mm} / \mathrm{in})$ - N core & NX & \\
\hline 20 & 175.5 & 180 & 47.6/1.9 (mm/in) - N core & NX & TD \\
\hline 1 & 0 & 28 & 47.6/1.9 (mm/in) - N core & NX & \\
\hline 2 & 28 & 40 & 47.6/1.9 (mm/in) - N core & NX & \\
\hline 3 & 40 & 56 & 47.6/1.9 (mm/in) - N core & NX & \\
\hline 4 & 56 & 66 & 47.6/1.9 (mm/in) - N core & NX & \\
\hline 5 & 66 & 75.5 & 47.6/1.9 (mm/in) - N core & NX & \\
\hline 6 & 75.5 & 85 & 47.6/1.9 (mm/in) - N core & NX & \\
\hline 7 & 85 & 94.5 & 47.6/1.9 (mm/in) - N core & NX & \\
\hline 8 & 94.5 & 103 & 47.6/1.9 (mm/in) - N core & NX & \\
\hline 9 & 103 & 112 & $47.6 / 1.9(\mathrm{~mm} / \mathrm{in})$ - N core & NX & \\
\hline 10 & 112 & 121.5 & $47.6 / 1.9(\mathrm{~mm} / \mathrm{in})-\mathrm{N}$ core & NX & \\
\hline 11 & 121.5 & 130.5 & 47.6/1.9 (mm/in) - N core & NX & \\
\hline 12 & 130.5 & 140.5 & 47.6/1.9 (mm/in) - N core & NX & \\
\hline 13 & 140.5 & 148 & $47.6 / 1.9(\mathrm{~mm} / \mathrm{in})-\mathrm{N}$ core & NX & TD \\
\hline 1 & 0 & 17 & $47.6 / 1.9(\mathrm{~mm} / \mathrm{in})$ - N core & NX & missing box \\
\hline 2 & 17 & 37.5 & 47.6/1.9 (mm/in) - N core & NX & \\
\hline 3 & 37.5 & 47 & 47.6/1.9 (mm/in) - N core & NX & \\
\hline 4 & 47 & 57 & 47.6/1.9 (mm/in) - N core & NX & \\
\hline 5 & 57 & 68 & 47.6/1.9 (mm/in) - N core & NX & \\
\hline 6 & 68 & 78.5 & 47.6/1.9 (mm/in) - N core & NX & \\
\hline 7 & 78.5 & 88 & $47.6 / 1.9(\mathrm{~mm} / \mathrm{in})$ - N core & NX & \\
\hline 8 & 88 & 99 & $47.6 / 1.9(\mathrm{~mm} / \mathrm{in})$ - N core & NX & \\
\hline 9 & 99 & 109 & 47.6/1.9 (mm/in) - N core & NX & \\
\hline
\end{tabular}




\section{Alaska GMC Russian Mountains Prospect Inventory}

Russian Mountains (Louise) Russian Mountains (Louise) Russian Mountains (Louise) Russian Mountains (Louise) Russian Mountains (Louise) Russian Mountains (Louise) Russian Mountains (Louise) Russian Mountains (Louise) Russian Mountains (Louise) Russian Mountains (Louise) Russian Mountains (Louise) Russian Mountains (Louise) Russian Mountains (Louise) Russian Mountains (Owhat) Russian Mountains (Owhat) Russian Mountains (Owhat) Russian Mountains (Owhat) Russian Mountains (Owhat) Russian Mountains (Owhat) Russian Mountains (Owhat) Russian Mountains (Owhat) Russian Mountains (Owhat) Russian Mountains (Owhat) Russian Mountains (Owhat) Russian Mountains (Owhat) Russian Mountains (Owhat) Russian Mountains (Owhat) Russian Mountains (Owhat) Russian Mountains (Owhat) Russian Mountains (Owhat) Russian Mountains (Owhat) Russian Mountains (Owhat) Russian Mountains (Owhat)

\begin{tabular}{|l|l} 
RM-011 & FMM \\
RM-011 & FMM \\
RM-011 & FMM \\
RM-011 & FMM \\
RM-011 & FMM \\
RM-011 & FMM \\
RM-011 & FMM \\
RM-011 & FMM \\
RM-011 & FMM \\
RM-011 & FMM \\
RM-011 & FMM \\
RM-011 & FMM \\
RM-011 & FMM \\
RM-012 & FMM \\
RM-012 & FMM \\
RM-012 & FMM \\
RM-012 & FMM \\
RM-012 & FMM \\
RM-012 & FMM \\
RM-012 & FMM \\
RM-012 & FMM \\
RM-012 & FMM \\
RM-012 & FMM \\
RM-012 & FMM \\
RM-012 & FMM \\
RM-012 & FMM \\
RM-012 & FMM \\
RM-012 & FMM \\
RM-012 & FMM \\
RM-012 & FMM \\
RM-012 & FMM \\
RM-012 & FMM \\
RM-012 & FMM \\
& \\
RMM
\end{tabular}

\begin{tabular}{|c|c|c|c|c|c|}
\hline 10 & 109 & 118 & 47.6/1.9 (mm/in) - N core & NX & \\
\hline 11 & 118 & 127.5 & 47.6/1.9 (mm/in) - N core & $N X$ & \\
\hline 12 & 127.5 & 136.5 & 47.6/1.9 (mm/in) - N core & NX & \\
\hline 13 & 136.5 & 145.5 & 47.6/1.9 (mm/in) - N core & $N X$ & \\
\hline 14 & 145.5 & 155 & 47.6/1.9 (mm/in) - N core & $N X$ & \\
\hline 15 & 155 & 165 & 47.6/1.9 (mm/in) - N core & $\mathrm{NX}$ & \\
\hline 16 & 165 & 174 & 47.6/1.9 (mm/in) - N core & $\mathrm{NX}$ & \\
\hline 17 & 174 & 183 & 47.6/1.9 (mm/in) - N core & $N X$ & \\
\hline 18 & 183 & 191.5 & 47.6/1.9 (mm/in) - N core & $N X$ & \\
\hline 19 & 191.5 & 201 & 47.6/1.9 (mm/in) - N core & $\mathrm{NX}$ & \\
\hline 20 & 201 & 210.5 & 47.6/1.9 (mm/in) - N core & $N X$ & \\
\hline 21 & 210.5 & 220 & 47.6/1.9 (mm/in) - N core & $\mathrm{NX}$ & \\
\hline 22 & 220 & 224 & 47.6/1.9 (mm/in) - N core & $\mathrm{NX}$ & TD \\
\hline 1 & 0 & 28 & 47.6/1.9 (mm/in) - N core & $N X$ & \\
\hline 2 & 28 & 46 & 47.6/1.9 (mm/in) - N core & $\mathrm{NX}$ & \\
\hline 3 & 46 & 61 & 47.6/1.9 (mm/in) - N core & $\mathrm{NX}$ & \\
\hline 4 & 61 & 71.5 & 47.6/1.9 (mm/in) - N core & $\mathrm{NX}$ & \\
\hline 5 & 71.5 & 81.5 & 47.6/1.9 (mm/in) - N core & $N X$ & \\
\hline 6 & 81.5 & 90 & 47.6/1.9 (mm/in) - N core & $\mathrm{NX}$ & \\
\hline 7 & 90 & 99 & 47.6/1.9 (mm/in) - N core & $\mathrm{NX}$ & \\
\hline 8 & 99 & 107.5 & 47.6/1.9 (mm/in) - N core & $N X$ & \\
\hline 9 & 107.5 & 116 & 47.6/1.9 (mm/in) - N core & $N X$ & \\
\hline 10 & 116 & 125 & 47.6/1.9 (mm/in) - N core & NX & \\
\hline 11 & 125 & 134 & 47.6/1.9 (mm/in) - N core & $\mathrm{NX}$ & \\
\hline 12 & 134 & 144 & $47.6 / 1.9$ (mm/in) - N core & $N X$ & \\
\hline 13 & 144 & 152.5 & 47.6/1.9 (mm/in) - N core & NX & \\
\hline 14 & 152.5 & 161 & 47.6/1.9 (mm/in) - N core & $\mathrm{NX}$ & \\
\hline 15 & 161 & 170 & 47.6/1.9 (mm/in) - N core & $N X$ & \\
\hline 16 & 170 & 179 & 47.6/1.9 (mm/in) - N core & $\mathrm{NX}$ & \\
\hline 17 & 179 & 188 & 47.6/1.9 (mm/in) - N core & $N X$ & \\
\hline 18 & 188 & 197.5 & $47.6 / 1.9$ (mm/in) - N core & $N X$ & \\
\hline 19 & 197.5 & 206 & 47.6/1.9 (mm/in) - N core & $\mathrm{NX}$ & \\
\hline 20 & 206 & 214.5 & 47.6/1.9 (mm/in) - N core & $N X$ & \\
\hline
\end{tabular}




\section{Alaska GMC Russian Mountains Prospect Inventory}

Russian Mountains (Owhat) Russian Mountains (Owhat) Russian Mountains (Owhat) Russian Mountains (Owhat) Russian Mountains (Owhat) Russian Mountains (Owhat) Russian Mountains (Owhat) Russian Mountains (Owhat) Russian Mountains (Owhat) Russian Mountains (Owhat) Russian Mountains (Owhat) Russian Mountains (Owhat) Russian Mountains (Owhat) Russian Mountains (Owhat) Russian Mountains (Owhat) Russian Mountains (Owhat) Russian Mountains (Owhat) Russian Mountains (Owhat) Russian Mountains (Owhat) Russian Mountains (Owhat) Russian Mountains (Owhat) Russian Mountains (Owhat) Russian Mountains (Owhat) Russian Mountains (Owhat) Russian Mountains (Owhat) Russian Mountains (Owhat) Russian Mountains (Owhat) Russian Mountains (Owhat) Russian Mountains (Owhat) Russian Mountains (Owhat) Russian Mountains (Owhat) Russian Mountains (Owhat) Russian Mountains (Owhat)

\begin{tabular}{ll|l} 
RM-012 & FMM \\
RM-012 & FMM \\
RM-012 & FMM \\
RM-012 & FMM \\
RM-012 & FMM \\
RM-012 & FMM \\
RM-012 & FMM \\
RM-012 & FMM \\
RM-012 & FMM \\
RM-012 & FMM \\
RM-012 & FMM \\
RM-012 & FMM \\
RM-012 & FMM \\
RM-012 & FMM \\
RM-012 & FMM \\
RM-013 & FMM \\
RM-013 & FMM \\
RM-013 & FMM \\
RM-013 & FMM \\
RM-013 & FMM \\
RM-013 & FMM \\
RM-013 & FMM \\
RM-013 & FMM \\
RM-013 & FMM \\
RM-013 & FMM \\
RM-013 & FMM \\
RM-013 & FMM \\
RM-013 & FMM \\
RM-013 & FMM \\
RM-013 & FMM \\
RM-013 & FMM \\
RM-013 & FMM \\
RM-013 & FMM \\
& \\
RMM
\end{tabular}

\begin{tabular}{|c|c|c|c|c|c|}
\hline 21 & 214.5 & 223.5 & 47.6/1.9 (mm/in) - $\mathrm{N}$ core & NX & \\
\hline 22 & 223.5 & 233.5 & 47.6/1.9 (mm/in) - $\mathrm{N}$ core & $N X$ & \\
\hline 23 & 233.5 & 243 & 47.6/1.9 (mm/in) - $\mathrm{N}$ core & NX & \\
\hline 24 & 243 & 252.5 & 47.6/1.9 (mm/in) - $\mathrm{N}$ core & $N X$ & \\
\hline 25 & 252.5 & 261.5 & $47.6 / 1.9(\mathrm{~mm} / \mathrm{in})-\mathrm{N}$ core & $\mathrm{NX}$ & \\
\hline 26 & 261.5 & 271 & $47.6 / 1.9$ (mm/in) - $\mathrm{N}$ core & $\mathrm{NX}$ & \\
\hline 27 & 271 & 280.5 & 47.6/1.9 (mm/in) - $\mathrm{N}$ core & $\mathrm{NX}$ & \\
\hline 28 & 280.5 & 290 & 47.6/1.9 (mm/in) - N core & $N X$ & \\
\hline 29 & 290 & 299 & 47.6/1.9 (mm/in) - N core & $N X$ & \\
\hline 30 & 299 & 308.5 & 47.6/1.9 (mm/in) - $\mathrm{N}$ core & $\mathrm{NX}$ & \\
\hline 31 & 308.5 & 317.5 & 47.6/1.9 (mm/in) - $\mathrm{N}$ core & $N X$ & \\
\hline 32 & 317.5 & 327 & 47.6/1.9 (mm/in) - N core & $\mathrm{NX}$ & \\
\hline 33 & 327 & 336.5 & 47.6/1.9 (mm/in) - $\mathrm{N}$ core & $\mathrm{NX}$ & \\
\hline 34 & 336.5 & 346 & 47.6/1.9 (mm/in) - N core & $N X$ & \\
\hline 35 & 346 & 351 & 47.6/1.9 (mm/in) - N core & $\mathrm{NX}$ & TD \\
\hline 1 & 5 & 15 & 47.6/1.9 (mm/in) - $\mathrm{N}$ core & $\mathrm{NX}$ & \\
\hline 2 & 15 & 29.5 & 47.6/1.9 (mm/in) - N core & $\mathrm{NX}$ & \\
\hline 3 & 29.5 & 39 & 47.6/1.9 (mm/in) - $\mathrm{N}$ core & $N X$ & \\
\hline 4 & 39 & 47.5 & 47.6/1.9 (mm/in) - $\mathrm{N}$ core & $\mathrm{NX}$ & \\
\hline 5 & 47.5 & 55.5 & 47.6/1.9 (mm/in) - N core & $\mathrm{NX}$ & \\
\hline 6 & 55.5 & 63.5 & 47.6/1.9 (mm/in) - $\mathrm{N}$ core & $N X$ & \\
\hline 7 & 63.5 & 73 & 47.6/1.9 (mm/in) - N core & $N X$ & \\
\hline 8 & 73 & 82.5 & 47.6/1.9 (mm/in) - $\mathrm{N}$ core & NX & \\
\hline 9 & 82.5 & 92 & 47.6/1.9 (mm/in) - $\mathrm{N}$ core & $\mathrm{NX}$ & \\
\hline 10 & 92 & 102.5 & $47.6 / 1.9(\mathrm{~mm} / \mathrm{in})$ - $\mathrm{N}$ core & $N X$ & \\
\hline 11 & 102.5 & 111.5 & 47.6/1.9 (mm/in) - N core & NX & \\
\hline 12 & 111.5 & 120.5 & 47.6/1.9 (mm/in) - $\mathrm{N}$ core & $\mathrm{NX}$ & \\
\hline 13 & 120.5 & 130 & 47.6/1.9 (mm/in) - N core & $N X$ & \\
\hline 14 & 130 & 139.5 & 47.6/1.9 (mm/in) - $\mathrm{N}$ core & $\mathrm{NX}$ & \\
\hline 15 & 139.5 & 140 & 47.6/1.9 (mm/in) - $\mathrm{N}$ core & $N X$ & \\
\hline 16 & 140 & 158 & $47.6 / 1.9$ (mm/in) - N core & $N X$ & \\
\hline 17 & 158 & 167.5 & 47.6/1.9 (mm/in) - $\mathrm{N}$ core & $\mathrm{NX}$ & \\
\hline 18 & 167.5 & 176.5 & 47.6/1.9 (mm/in) - $\mathrm{N}$ core & $N X$ & \\
\hline
\end{tabular}




\section{Alaska GMC Russian Mountains Prospect Inventory}

Russian Mountains (Owhat) Russian Mountains (Owhat) Russian Mountains (Owhat) Russian Mountains (Owhat) Russian Mountains (Owhat) Russian Mountains (Owhat) Russian Mountains (Owhat) Russian Mountains (Owhat) Russian Mountains (Owhat) Russian Mountains (Owhat) Russian Mountains (Owhat) Russian Mountains (Owhat) Russian Mountains (Owhat) Russian Mountains (Owhat) Russian Mountains (Owhat) Russian Mountains (Owhat) Russian Mountains (Owhat) Russian Mountains (Owhat) Russian Mountains (Owhat) Russian Mountains (Owhat) Russian Mountains (Owhat) Russian Mountains (Owhat) Russian Mountains (Owhat) Russian Mountains (Owhat) Russian Mountains (Owhat) Russian Mountains (Owhat) Russian Mountains (Owhat) Russian Mountains (Owhat) Russian Mountains (Owhat) Russian Mountains (Owhat) Russian Mountains (Owhat) Russian Mountains (Owhat) Russian Mountains (Owhat)

\begin{tabular}{|l|l} 
RM-013 & FMM \\
RM-013 & FMM \\
RM-013 & FMM \\
RM-013 & FMM \\
RM-013 & FMM \\
RM-013 & FMM \\
RM-013 & FMM \\
RM-013 & FMM \\
RM-013 & FMM \\
RM-013 & FMM \\
RM-013 & FMM \\
RM-013 & FMM \\
RM-013 & FMM \\
RM-013 & FMM \\
RM-013 & FMM \\
RM-013 & FMM \\
RM-013 & FMM \\
RM-013 & FMM \\
RM-013 & FMM \\
RM-013 & FMM \\
RM-013 & FMM \\
RM-013 & FMM \\
RM-013 & FMM \\
RM-013 & FMM \\
RM-013 & FMM \\
RM-013 & FMM \\
RM-013 & FMM \\
RM-013 & FMM \\
RM-013 & FMM \\
RM-013 & FMM \\
RM-013 & FMM \\
RM-013 & FMM \\
RM-013 & FMM \\
& \\
RMM
\end{tabular}

\begin{tabular}{|c|c|c|c|c|c|}
\hline 19 & 176.5 & 186 & 47.6/1.9 (mm/in) - N core & $\mathrm{NX}$ & \\
\hline 20 & 186 & 196 & $47.6 / 1.9(\mathrm{~mm} / \mathrm{in})$ - N core & $\mathrm{NX}$ & \\
\hline 21 & 196 & 204 & $47.6 / 1.9(\mathrm{~mm} / \mathrm{in})$ - $\mathrm{N}$ core & $\mathrm{NX}$ & \\
\hline 22 & 204 & 214.5 & $47.6 / 1.9(\mathrm{~mm} / \mathrm{in})$ - N core & NX & \\
\hline 23 & 214.5 & 224 & $47.6 / 1.9(\mathrm{~mm} / \mathrm{in})$ - N core & $\mathrm{NX}$ & \\
\hline 24 & 224 & 233.5 & $47.6 / 1.9(\mathrm{~mm} / \mathrm{in})$ - $\mathrm{N}$ core & $\mathrm{NX}$ & \\
\hline 25 & 233.5 & 242.5 & $47.6 / 1.9(\mathrm{~mm} / \mathrm{in})$ - N core & NX & \\
\hline 26 & 242.5 & 252 & $47.6 / 1.9(\mathrm{~mm} / \mathrm{in})$ - $\mathrm{N}$ core & $\mathrm{NX}$ & \\
\hline 27 & 252 & 261.5 & $47.6 / 1.9(\mathrm{~mm} / \mathrm{in})$ - $\mathrm{N}$ core & NX & \\
\hline 28 & 261.5 & 270.5 & $47.6 / 1.9(\mathrm{~mm} / \mathrm{in})$ - N core & $\mathrm{NX}$ & \\
\hline 29 & 270.5 & 280 & $47.6 / 1.9(\mathrm{~mm} / \mathrm{in})$ - $\mathrm{N}$ core & $\mathrm{NX}$ & \\
\hline 30 & 280 & 289.5 & 47.6/1.9 (mm/in) - N core & NX & \\
\hline 31 & 289.5 & 298 & $47.6 / 1.9(\mathrm{~mm} / \mathrm{in})$ - N core & $\mathrm{NX}$ & \\
\hline 32 & 298 & 307.5 & $47.6 / 1.9(\mathrm{~mm} / \mathrm{in})$ - $\mathrm{N}$ core & $\mathrm{NX}$ & \\
\hline 33 & 307.5 & 316.5 & 47.6/1.9 (mm/in) - N core & $\mathrm{NX}$ & \\
\hline 34 & 316.5 & 325 & $47.6 / 1.9(\mathrm{~mm} / \mathrm{in})$ - N core & $\mathrm{NX}$ & \\
\hline 35 & 325 & 334.5 & 47.6/1.9 (mm/in) - N core & $\mathrm{NX}$ & \\
\hline 36 & 334.5 & 343 & $47.6 / 1.9(\mathrm{~mm} / \mathrm{in})$ - N core & $\mathrm{NX}$ & \\
\hline 37 & 343 & 353 & $47.6 / 1.9(\mathrm{~mm} / \mathrm{in})$ - N core & $\mathrm{NX}$ & \\
\hline 38 & 353 & 361.5 & 47.6/1.9 (mm/in) - N core & $\mathrm{NX}$ & \\
\hline 39 & 361.5 & 371 & $47.6 / 1.9(\mathrm{~mm} / \mathrm{in})$ - N core & $\mathrm{NX}$ & \\
\hline 40 & 371 & 380 & 47.6/1.9 (mm/in) - N core & $\mathrm{NX}$ & \\
\hline 41 & 380 & 389 & 47.6/1.9 (mm/in) - N core & NX & \\
\hline 42 & 389 & 399 & $47.6 / 1.9(\mathrm{~mm} / \mathrm{in})$ - N core & $\mathrm{NX}$ & \\
\hline 43 & 399 & 408 & $47.6 / 1.9(\mathrm{~mm} / \mathrm{in})$ - $\mathrm{N}$ core & $\mathrm{NX}$ & \\
\hline 44 & 408 & 418 & $47.6 / 1.9(\mathrm{~mm} / \mathrm{in})$ - $\mathrm{N}$ core & $\mathrm{NX}$ & \\
\hline 45 & 418 & 426.5 & $47.6 / 1.9(\mathrm{~mm} / \mathrm{in})$ - N core & $\mathrm{NX}$ & \\
\hline 46 & 426.5 & 436 & $47.6 / 1.9(\mathrm{~mm} / \mathrm{in})$ - N core & $\mathrm{NX}$ & \\
\hline 47 & 436 & 445.5 & 47.6/1.9 (mm/in) - N core & $\mathrm{NX}$ & \\
\hline 48 & 445.5 & 455 & $47.6 / 1.9(\mathrm{~mm} / \mathrm{in})$ - N core & $\mathrm{NX}$ & \\
\hline 49 & 455 & 465 & $47.6 / 1.9(\mathrm{~mm} / \mathrm{in})$ - $\mathrm{N}$ core & $\mathrm{NX}$ & \\
\hline 50 & 465 & 474.5 & $47.6 / 1.9(\mathrm{~mm} / \mathrm{in})$ - $\mathrm{N}$ core & $\mathrm{NX}$ & \\
\hline 51 & 474.5 & 484 & $47.6 / 1.9(\mathrm{~mm} / \mathrm{in})$ - N core & $N X$ & \\
\hline
\end{tabular}




\section{Alaska GMC Russian Mountains Prospect Inventory}

\begin{tabular}{l|l|l}
\hline Russian Mountains (Owhat) & RM-013 & FMM \\
\hline Russian Mountains (Owhat) & RM-013 & FMM \\
\hline Russian Mountains (Owhat) & RM-013 & FMM \\
\hline Russian Mountains (Owhat) & RM-013 & FMM \\
\hline Russian Mountains (Owhat) & RM-013 & FMM \\
\hline Russian Mountains (Owhat) & RM-013 & FMM \\
Russian Mountains (Owhat) & RM-013 & FMM \\
\hline Russian Mountains (Owhat) & RM-013 & FMM \\
\hline Russian Mountains (Owhat) & RM-013 & FMM \\
\hline Russian Mountains (Owhat) & RM-013 & FMM \\
\hline Russian Mountains (Owhat) & RM-013 & FMM \\
\hline Russian Mountains (Owhat) & RM-013 & FMM \\
\hline Russian Mountains (Owhat) & RM-013 & FMM \\
\hline Russian Mountains (Owhat) & RM-013 & FMM
\end{tabular}

\begin{tabular}{|c|c|c|c|c|c|}
\hline 52 & 484 & 493.5 & 47.6/1.9 (mm/in) - N core & NX & \\
\hline 53[ & 493.5 & 502.5 & 47.6/1.9 (mm/in) - N core & $N X$ & \\
\hline 54 & 502.5 & 512 & 47.6/1.9 (mm/in) - N core & NX & \\
\hline 55 & 512 & 521.5 & 47.6/1.9 (mm/in) - N core & $\mathrm{NX}$ & \\
\hline 56 & 521.5 & 530 & 47.6/1.9 (mm/in) - N core & NX & \\
\hline 57 & 530 & 539.5 & 47.6/1.9 (mm/in) - N core & NX & \\
\hline 58 & 539.5 & 548.5 & 47.6/1.9 (mm/in) - N core & NX & \\
\hline 59 & 548.5 & 558 & 47.6/1.9 (mm/in) - N core & $\mathrm{NX}$ & \\
\hline 60 & 558 & 567 & 47.6/1.9 (mm/in) - N core & $\mathrm{NX}$ & \\
\hline 61 & 567 & 577.5 & 47.6/1.9 (mm/in) - N core & $\mathrm{NX}$ & \\
\hline 62 & 577.5 & 587 & 47.6/1.9 (mm/in) - N core & NX & \\
\hline 63 & 587 & 596.5 & 47.6/1.9 (mm/in) - N core & NX & \\
\hline 64 & 596.5 & 605 & 47.6/1.9 (mm/in) - N core & $\mathrm{NX}$ & \\
\hline 65 & 605 & 610 & 47.6/1.9 (mm/in) - N core & $N X$ & TD \\
\hline
\end{tabular}

